Editorial

\title{
Acknowledgement to Reviewers of Risks in 2018
}

\section{Risks Editorial Office}

MDPI, St. Alban-Anlage 66, 4052 Basel, Switzerland

Published: 8 January 2019

Rigorous peer-review is the corner-stone of high-quality academic publishing. The editorial team greatly appreciates the reviewers who contributed their knowledge and expertise to the journal's editorial process over the past 12 months. In 2018, a total of 145 papers were published in the journal, with a median time to first decision of 20.7 days and a median time to publication of 43.9 days. The editors would like to express their sincere gratitude to the following reviewers for their cooperation and dedication in 2018:

Adenusi, Joachim

Aguilar, Jean-Philippe

Ali Nasir, Muhammad

Alzaghal, Ahmaed

Amezcua, Javier

Amores-Salvadó, Javier

Andreeva, Galina

Angelini, Eliana

Anthropelos, Michail

Antoine, Bertille

Anton, Sorin

Antonio, Di Crescenzo

Arciénaga Morales, Antonio Adrián

Arnold, Séverine

Atukeren, Erdal

Audrino, Francesco

Avraam, Demetris

Avram, Florin

Awudu, Iddrisu

Ayuso, Mercedes

Baek, Seungho

Baker, Tim

Balbás, Alejandro

Baldauf, Michael

Ballestra, Luca Vincenzo

Bardoutsos, Anastasios

Bariviera, Aurelio F.

Basei, Matteo

Bavoso, Vincenzo

Beg, Rabiul

Belak, Christoph

Błach, Joanna

Boguslavskaya, Elena
Boj Del Val, Eva

Bolance, Catalina

Borochkin, Alexander

Bostan, Ionel

Boubaker, Sabri

Buchner, Axel

Caines, Peter E.

Calderin, Enrique

Callegaro, Giorgia

Caporin, Massimiliano

Caraiani, Petre

Chaigneau, Pierre

Chang, Chia-Lin

Charles-Cadogan, G.

Chatrath, Mr. Suraj

Chaudhury, Mo

Chen, Chun-Da

Chen, James M.

Chen, Ying-Ju

Chen, Yiqing

Cheng, Jiuqing

Cheng, Po-Keng

Cheridito, Patrick

Cheung, Eric

Chiappini, Helen

Chileshe, Nicholas

Chow, Victor

Chowdhury, Jaideep

Choy, Boris

Christensen, Sören

Claramunt, Mercedes

Clemente, Gianpaolo

Cohen, Albert 
Coleman, Thomas F.

Consiglio, Andrea

Consiglio, Andrea

Cooray, Kahadawala

Cui, Zhenyu

Damjanovic, Vladislav

Dassios, Angelos

De Angelis, Luca

De Giuli, Maria Elena

Delcea, Camelia

Devolder, Pierre

Di Lorenzo, Emilia

Dixon, Matthew F.

Donkor, Emmanuel

Donnelly, Ryan

Dungey, Mardi

Dvouletý, Ondřej

Egedorf, Maren

Egidio Dos Reis, Alfredo

Ender, Manuela

Eves, Chris

Fanelli, Viviana

Fedotenkov, Igor

Feinstein, Zachary

Feldman, David

Feng, Runhuan

Feng, Shih-Ping

Ferreira, Paulo

Fiedler, Ingo

Filipovic, Damir

Fiori, Anna Maria

Floreani, Josanco

Fontana, Claudio

Forero-Laverde, Germán

Foscolo, Enrico

Fouque, Jean-Pierre

Fry, John

Fujita, Toshiyuki

Fuller, David L.

Furmańczyk, Konrad

Fusari, Nicola

Fusco, Giulio

Gan, Guojun

Garcia, Jennifer Alonso

Gavriletea, Marius

Georgopoulou, Elena

Gherghina, Ştefan Cristian

Ghosh, Subir

Giesecke, Kay

Glabadanidis, Paskalis

Goerlandt, Floris

Gómez-Déniz, Emilio
Grazian, Clara

Grima, Simon

Guasoni, Paolo

Guharay, Sabyasachi

Guijarro, Francisco

Guillén, Montserrat

Hamori, Shigeyuki

Hanewald, Katja

Hanson, Floyd B.

Harrison, Glenn

Hashorva, Enkelejd

Hassani, Bertrand

Heo, Wookjae

Hertrich, Markus

Herzog, Bodo

Hiabu, Munir

Hieber, Peter

Högberg, Lovisa

$\mathrm{Hsu}$, Ming-Fu

Ingrassia, Salvatore

Ivanov, Eugen

Jevtic, Petar

Jiang, Wenjun

Jin, Can

Jonk, Yvonne

Kaishev, Vladimir K.

Keilman, Nico W.

Kennett, Ron

Kermarrec, Gael

Khemka, Gaurav

Kim, Jong-Min

Kim, Seong Dae

Kinateder, Harald

Kiseláková, Dana

Kitapbayev, Yerkin

Kolkiewicz, Adam W.

Koller, Michael

Konstantinides, Dimitrios

Koraz, Yahya

Korbel, Jan

Koulouriotis, Dimitrios E.

Kumar, Kuldeep

Kyriazis, Nikolaos A.

Lakner, Peter

Land, Kenneth C.

Laurila, Hannu

Lazar, Emese

Lee, Cheng-Ming

Lee, Gee

Li, Frank

Li, Jackie

Li, Shuanming 
Li, Xiaofei

Lim, Byung Hwa

Lim, Dongkuk

Lin, Tzuling

Lioukas, Spyros

Liu, Liqun

Liu, Rui

Low, Rand

Luo, Shangzhen

Lyhagen, Johan

Magistris, Paolo Santucci De

Mailhot, Mélina

Maller, Ross

Manamperi, Nimantha

Manganelli, Benedetto

Manta, Otilia

Mantovani, Marco

Marazzina, Daniele

Marchesi, Michele

Marques, Rui

Masud, Md. Abdul Kaium

Mazzù, Sebastiano

Menoncin, Francesco

Michael Curran, Michael

Mikayilov, Jeyhun I

Mildenhall, Stephen

Milena, Jakšić

Miljkovic, Tatjana

Miller, Kirk

Minca, Andreea

Mizgier, Kamil

Modica, Salvatore

Moenig, Thorsten

Molina Serrano, Beatriz

Moretto, Enrico

Morley, Bruce

Moutanabbir, Khouzeima

Munir, Rahat

Murphy, Austin

Nakajima, Jouchi

Narayan, Seema

Németh, László

Nguyen, T.Thanh-Binh

Nielsen, Bent

Nielsen, Heino Bohn

Nielsen, Jens Perch

Nunes, Cláudia

Nwafor, Chioma

Oglend, Atle

Okhrati, Ramin

Olenko, Andriy

Oncioiu, Ionica
O'neill, Eoin

O'neill, Robert

Oosterlee, Kees

Ortega, Jose Antonio

Ortiz-Latorre, Salvador

Ortobelli, Sergio

Pacelli, Graziella

Pantelous, Athanasios

Paolella, Marc S.

Park, Chanseok

Parker, Thomas

Peerenboom, Peter Bob

Perote, Javier

Pesta, Michal

Peter, Richard

Peters, Gareth

Pié, Laia

Pirjan, Alexandru

Pirra, Marco

Plakandaras, Vasilios

Porzio, Claudio

Poulsen, Rolf

Powell, Robert J.

Powers, Michael R

Provost, Serge

Reimers, Hans-Eggert

Righi, Simone

Rodosthenous, Neofytos

Rodrigo-González, Amalia

Rosato, Paolo

Rosazza Gianin, Emanuela

Rostam-Afschar, Davud

Russolillo, Maria

Salas, Jesus M.

Salhi, Yahia

Salvador, Manuel

Salvioni, Daniela

Samanthi, Ranadeera

Samitas, Aristeidis

Santoboni, Fabrizio

Sawik, Bartosz

Scaillet, Olivier

Schlögl, Erik

Schlütter, Sebastian

Schmidt, Klaus D.

Scotti, Simone

Sendova, Kristina

SenGupta, Indranil

Serrano González, Javier

Shen, Yang

Sherris, Michael

Shushi, Tomer 
Silvestrov, Dmitrii

Sirghi, Nicoleta

Škrinjarić, Tihana

Sonenshine, Ralph

Stejskal, Jan

Strozzi, Fernanda

$\mathrm{Su}$, Jianxi

Swinkels, Laurens

Swishchuk, Anatoliy

Syriopoulos, Konstantinos

Szimayer, Alexander

Takahashi, Satoru

Takahashi, Taiki

Tang, Jian

Tarrant, Wayne

Tchatoka, Firmin Doko

Thøgersen, Julie

Tian, Ruilin

Tian, Weidong

Tsai, I-Chun

Tsiropoulou, Eirini Eleni

Tunaru, Radu S.

Valdez, Emiliano A.

Vanduffel, Steven

Vetzal, Ken

Villegas, Andrés

Vitali, Sebastiano

Vouldis, Angelos T.

Vuong, Quan-Hoang

Wakker, Peter
Walker, Patrick

Walther, Thomas

Wang, Deshen

Wang, Min

Wang, Ruodu

Wang, Yang

Weng, Chengguo

West, Jason

Westerholm, Joakim

Wieczorek-Kosmala, Monika

Wölfle, Marco

Xu, Lei

$\mathrm{Xu}$, Mengyi

Yamazaki, Kazutoshi

Yang, Hailiang

Yang, Lu

Yang, Yipeng

Younis, Mustafa Z.

Yu, Daoping

Zagaglia, Paolo

Zanón, José Luis Vilar

Zeitsch, Peter J.

Zhang, Xibin

Zhao, Tian

Zhou, Xiaowen

Zhu, Lingjiong

Zhuang, Shengchao

Zitkovic, Gordan

(C) 2019 by the author. Licensee MDPI, Basel, Switzerland. This article is an open access article distributed under the terms and conditions of the Creative Commons Attribution (CC BY) license (http://creativecommons.org/licenses/by/4.0/). 